\title{
Tumour-Immune Interaction Model with Cell Cycle Effects Including $G_{0}$ Phase
}

\author{
${ }^{1}$ Nor Aziran Awang, ${ }^{2}$ Normah Maan* and ${ }^{3}$ Dasuki Sul'ain \\ ${ }^{1,2}$ Department of Mathematical Sciences, Faculty of Science \\ Universiti Teknologi Malaysia, 81310 Johor Bahru, Johor, Malaysia \\ ${ }^{3}$ School of Health Sciences, \\ Universiti Sains Malaysia, 16150 Kubang Kerian, Kelantan, Malaysia \\ ${ }^{*}$ Corresponding author: normahmaan@utm.my
}

Article history

Received: 30 September 2018

Received in revised form: 6 December 2018

Accepted: 17 December 2018

Published on line: 31 December 2018

\begin{abstract}
Tumour cells behave differently than normal cells in the body. They grow and divide in an uncontrolled manner (actively proliferating) and fail to respond to signal. However, there are cells that become inactive and reside in quiescent phase $\left(G_{0}\right)$. These cells are known as quiescence cells that are less sensitive to drug treatments (radiotherapy and chemotherapy) than actively proliferation cells. This paper proposes a new mathematical model that describes the interaction of tumour growth and immune response by considering tumour population that is divided into three different phases namely interphase, mitosis and $G_{0}$. The model consists of a system of delay differential equations where the delay, $\tau$ represents the time for tumour cell to reside interphase before entering mitosis phase. Stability analysis of the equilibrium points of the system was performed to determine the dynamics behaviour of system. Result showed that the tumour population depends on number of tumour cells that enter active (interphase and mitosis) and $G_{0}$ phases. This study is important for treatment planning since tumour cell can resist treatment when they refuge in a quiescent state.
\end{abstract}

Keywords Tumour growth; immune system; cell cycle; quiescent phase normalization.

Mathematics Subject Classification 93A30, 92B05, 93D99.

\section{Introduction}

Tumour is one of the most dangerous and complicated illnesses causing death every year [12]. It occurs when the cell cycle failed to control the proliferation of the cells. These extra cells can continuously divide and may form growth called tumour. Tumour cells may able to ignore signals that normally tell the cells to stop dividing. The treatments are still difficult to find. Chemotherapy, radiotherapy and surgery are introduced as traditional treatments and have been recorded to be partially successful since these treatments also affect the normal cells [3-4]. Researchers keep looking for other treatments to improve the available treatments. 
Then, immunotherapy is introduced [3-8], which is a treatment based on immune system. Immune system is a network of cells, organs, tissues and proteins that protect the body against infections and other invaders. It is a complex system in the human body. It involves many biological structures and processes to fight diseases. The system normally monitor all the substances found in the body. Then, it automatically raises alarm and attacks when there are new unrecognised substances. Researches show that tumour cells can be overcome by different types of immune cells where each cell has its own role in killing tumour cells [8-12]. d'Onofrio [9] introduced a mathematical model that gives a general framework of tumour-immune interaction and followed by other researchers [3-5,8-11]. However, most of the tumour-immune models use logistic function, gompertz and exponential function as the growth term $[3,4,8,9]$.

Villasana [13] proposed mitosis as the growth term in tumour population. She introduced a tumour-immune system model that involved cell-cycle. The population of tumour took place during interphase and mitosis ( $\mathrm{M}$ phase). However, Villasana only considered an active phase of cell cycle without considering inactive phase. A follow up study of Villasana's model was recently conducted by Liu [14]. The author modified the model by adding quiescent phase into the model. The results showed that the resting phase (quiescent phase) was the most important compartment for tumour treatment. The concern of this study is to use Liu's model by considering the cells from mitosis that will enter either mitosis phase or quiescent phase compared to Liu that considered cells from mitosis entering the quiescent phase. This work discusses on Delay Differential Equations (DDEs).

\section{The Model}

Cell cycle is a cycle of a cell from original cell that divides into two new cells. It involves multiple checkpoints that excess multiple processes; signals, cell size and DNA integrity. The cell cycle is divided into two major phases, interphase and mitosis. During interphase, the cell grows and makes a copy of DNA. The phase consists of $G_{1}, \mathrm{~S}$ and $G_{2}$ phases. $G_{1}$ phase also known as pre-synthesis phase is the first phase where cell prepares to growth. Then, the cell enters $\mathrm{S}$ phase (synthesis phase) to complete the replication of DNA. This is followed by $G_{2}$ phase, the second gap phase, where the cell grows more and begins the preparation for mitosis. $G_{2}$ phase ends when mitosis begins. After the M-phase (mitosis) completed, two new daughter cells enter $G_{1}$ phase and continue the cycle (Figure 1). $G_{1}$ phase is the longest phase in the cell cycle. It could last as long as 48 hours. Therefore, a time delay was added into the present model to represent the cells residing the inter phase.

However, there are cells that enter $G_{0}$ phase and live in a quiescent state [14]. In $G_{0}$ phase, the cell is not actively ready to divide. The cell will become active after receiving the right signals. In cancer treatment, the cells that enter $G_{0}$ phase are called as quiescent cells. Quiescent cell can avoid the treatment in the resting phase and its maintain survive after a successful treatment. The surviving quiescent cell can continue the growth of tumour when treatment effect has failed [14]. Therefore, the $G_{0}$ phase is an important phase that needs to be understood in scheduling cancer treatment.

The presented model was based on the model by Liu et al. [14]. In the model, the interaction of tumour cell and immune cell was proposed. The basic idea of the model is that a tumour consists of cells that are actively proliferating and those that are quiescent. Proliferating cell is an active part that undergo mitosis (dividing) while quiescent cell is clarified as an inactive 


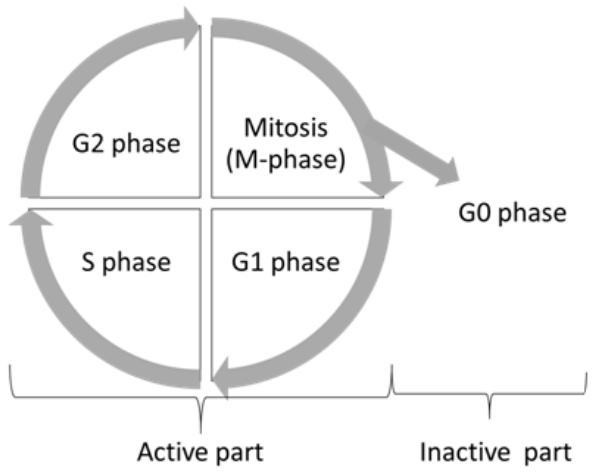

Figure 1: Cell Cycle Phases

cell and does not have enough nutrients for cell growth and division. After a certain time, the quiescent cell will enter the active phase and start the cycle again. In Liu, it was assumed that all newly generated daughter cells will directly enter the quiescent phase. In the proposed model, the assumption was loosen, which allow generated cells to be in either active or quiescent phase [15].

Immune system is a complicated system. There are many types of immune cell with different functions in eliminating the tumour cells $[6-7,16]$. One of the important cells is lymphocyte. Lymphocyte is one of several different types of white blood cells. It consists of innate and adaptive immunity. They have different roles and ability to fight tumour cells. Innate immunity involves macrophages, natural killer (NK) cells and dendritic cells while adaptive immunity can involve T and B lymphocytes. However, in this model, the focus was not on any specific types of lymphocytes. It was generally assumed that there exist lymphocytes that interact with tumour cell at any phase of the cell. All the interactions are depicted in the schematic diagram in Figure 2. Term $2 a_{3}(1-f)$ and $2 a_{3} f$ which are represent the cell from mitosis that enter inter phase and quiescent phase, are introduced as the new assumptions.

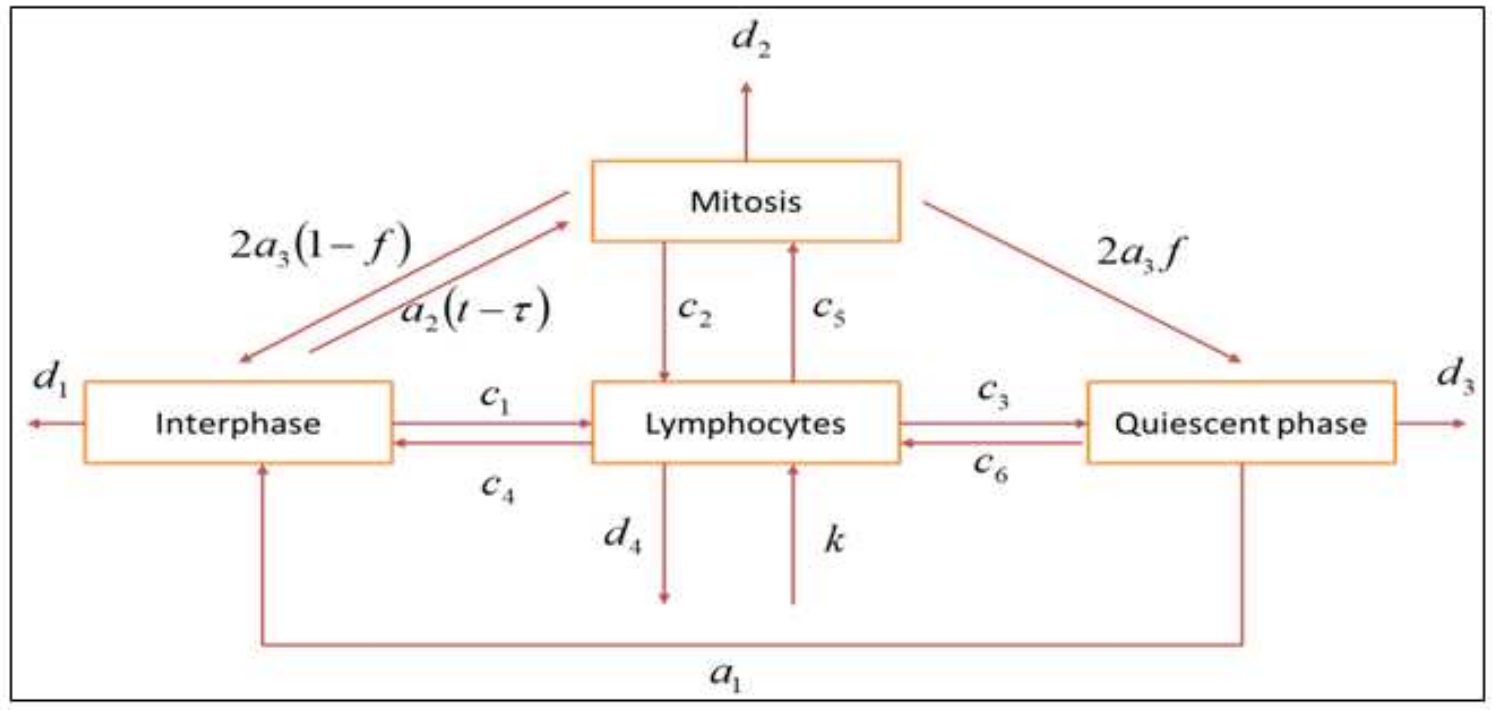

Figure 2: Schematic Diagram for Tumour-Immune Interaction Model with Cell Cycle Effect

In this paper, based on the model of Liu [14] and followed by new assumptions, a delayed 
model of tumour-immune system interaction was considered according to the following form

$$
\begin{aligned}
& x(t)=\underbrace{a_{1} z(t)}_{\text {from resting phase }}+\underbrace{2 a_{3}(1-f) y(t)}_{\text {from mitosis phase }}-\underbrace{a_{2} x(t)}_{\text {flowing into mitosis phase }}-\underbrace{d_{1} x(t)}_{\text {natural death }} \\
& \text { - } \underbrace{c_{1} x(t) I(t)}_{\text {destroyed by lymphocytes }}, \\
& y(t)=\underbrace{a_{2} x(t-\tau)}_{\text {from interphase }}-\underbrace{a_{3} y(t)}_{\text {flowing into mitosis phase }}-\underbrace{d_{2} y(t)}_{\text {natural death }}-\underbrace{c_{2} y(t) I(t)}_{\text {destroyed by lymphocyes }}, \\
& z(t)=\underbrace{2 a_{3} f y(t)}_{\text {from mitosis phase }}-\underbrace{a_{1} z(t)}_{\text {flowing into interphase }}-\underbrace{d_{3} z(t)}_{\text {natural death }}-\underbrace{c_{3} z(t) I(t)}_{\text {destroyed by lymphocyes }}, \\
& I(t)=\underbrace{k}_{\text {constant growth source }}-\underbrace{\frac{\rho I(t)(x+y+z)^{n}}{\alpha+(x+y+z)^{n}}}_{\text {growth due to stimulus }}-\underbrace{d_{4} I(t)}_{\text {natural death }}-\underbrace{c_{4} x(t) I(t)}_{\text {destroyed by tumour in interphase }} \\
& -\underbrace{c_{5} y(t) I(t)}_{\text {destroyed by tumour in mitosis phase }}-\underbrace{c_{6} z(t) I(t)}_{\text {destroyed by tumour in resting phase }} .
\end{aligned}
$$

$x(t), y(t)$ and $z(t)$ are the population of tumour during interphase, mitosis and quiescent phases; $I(t)$ is the population of lymphocytes at time $t$, respectively. All constants were positive and interpreted as follow:

- Term $a_{i} \quad(i=1,2,3)$ represent the proliferation rate of different stages of cell cycle; $c_{i}(i=1,2,3, \ldots, 6)$ terms represent the number of immune cells that become inactive due to attack of tumour cells [13-14].

- Natural death rate of cell was represented by the terms $d_{1} x(t), d_{2} y(t), d_{3} z(t)$ and $d_{4} I(t)$ [13-14].

- Term

$$
\frac{\rho I(t)(x+y+z)^{n}}{\alpha+(x+y+z)^{n}}
$$

represents the nonlinear growth of the immune population due to stimulus by tumour cells. Michaelis- Menten form were chosen for this term following others [9,13-14].

- The term $\rho$ represents the increase of immune cells due to stimulus and $\alpha$ represents the half value for the immune response. $n$ represents time for immune system to detect the tumour cell [13-14].

- $f$ represents the population of tumour cells that enter quiescent phase, $(0<f \leq 1)$

- $\tau$ represents time for tumour cell reside in interphase before entering mitosis. Thus term $x(t-\tau)$ has introduced in the system. [14].

\section{$3 \quad$ Stability Analysis}

It is important to look at the long-term behaviour of tumour cell populations. This study began to determine the dynamics of the system without immune response followed by the system with immune response. 


\subsection{Tumour-Model in the Absence of Immune Response}

In the absence of immune response, the system was presented as

$$
\begin{aligned}
& x(t)=a_{1} z(t)+2 a_{3}(1-f) y(t)-a_{3} x(t)-d_{1} x(t), \\
& y(t)=a_{2} x(t-\tau)-a_{3} y(t)-d_{2} y(t), \\
& z(t)=2 a_{3} f y(t)-a_{1} z(t)-d_{3} z(t) .
\end{aligned}
$$

The only equilibrium point for this system was $E_{0}=(0,0,0)$. The system was linearized around the fixed point and produced

$$
A=\left[\begin{array}{ccc}
-a_{2}-d_{1} & 2 a_{3}(1-f) & a_{1} \\
0 & -a_{3}-d_{2} & 0 \\
0 & 2 a_{3} f & -a_{1}-d_{3}
\end{array}\right], B=e^{-\lambda \tau}\left[\begin{array}{lll}
0 & 0 & 0 \\
a_{2} & 0 & 0 \\
0 & 0 & 0
\end{array}\right]
$$

and the characteristic equation became

$$
\lambda^{3}+A_{1} \lambda^{2}+A_{2} \lambda+A_{3}+\left(B_{1} \lambda+B_{2}\right) e^{-\lambda \tau}=0
$$

where

$$
\begin{aligned}
& A_{1}=a_{1}+a_{2}+a_{3}+d_{1}+d_{2}+d_{3}, \\
& A_{2}=a_{1} a_{2}+a_{1} a_{3}+a_{2} a_{3}+a_{1} d_{1}+a_{3} d_{1}+a_{1} d_{2}+a_{2} d_{2}+d_{1} d_{2}+a_{2} a_{3}+a_{3} d_{3}+d_{1} d_{3}+d_{2} d_{3}, \\
& A_{3}=a_{1} a_{2} a_{3}+a_{1} a_{3} d_{1}+a_{1} a_{2} d_{2}+a_{1} d_{1} d_{2}+a_{2} a_{3} d_{3}+a_{3} d_{1} d_{3}+a_{2} d_{2} d_{3}+d_{1} d_{2} d_{3}, \\
& B_{1}=-2(1-f) a_{2} a_{3} \\
& B_{2}=-2(1-f) a_{1} a_{2} a_{3}-2(1-f) a_{2} a_{3} d_{3}-2 f a_{1} a_{2} a_{3} .
\end{aligned}
$$

For the case of $\tau=0$, the characteristic equation was

$$
\lambda^{3}+A_{1} \lambda^{2}+\left(A_{2}+B_{1}\right) \lambda+\left(A_{3}+B_{2}\right)=0 .
$$

By Routh-Hurwitz Criteria, all roots of the characteristic Equation (3) have negative real part if and only if

1. $A_{1}>0$.

2. $A_{3}+B_{2}>0$.

3. $A_{1}\left(A_{2}+B_{1}\right)>\left(A_{3}+B_{2}\right)$.

Now, the case $\tau>0$ was considered. The characteristic equation was recalled as in (2). Let $\sigma>0$ and suppose $\lambda>i \sigma$ is a pure imaginary root of characteristic equation

$$
\begin{aligned}
& -\left(-A_{1} \sigma^{2}+A_{3}\right)=B_{2} \cos (\sigma \tau)+B_{1} \sigma \sin (\sigma \tau), \\
& \left(-\sigma^{3}+A_{2} \sigma\right)=B_{2} \sin (\sigma \tau)-B_{1} \sigma \cos (\sigma \tau) .
\end{aligned}
$$

Squaring and adding both equations led to

$$
\mu^{3}+\alpha_{1} \mu^{2}+\alpha_{2} \mu+\alpha_{3}=0, \quad\left(\mu=\sigma^{2}\right)
$$


where

$$
\alpha_{1}=A_{1}+2 A_{2}, \quad \alpha_{2}=A_{2}-2 A_{1} A_{3}-B_{1}^{2}, \quad \alpha_{3}=A_{3}^{2}-B_{2}^{2} .
$$

As a result, the delay term and trigonometric term of the polynomial equation disappeared. This study key only interested in the case when $\sigma \in \Re$. Suppose that the characteristic equation (5) has a positive real root defined by $\mu^{*}$. Solving both equations (4) with delay $\tau$ corresponding to $\sigma^{*}= \pm \sqrt{\mu^{*}}$ yielded the critical value $\tau_{0}$

$$
\tau_{0}=\frac{1}{\sigma}\left\{\frac{-\left(B_{2}\right)\left(-A_{1} \sigma^{2}+A_{3}\right)-\left(-\sigma^{3}+A_{2} \sigma\right)\left(B_{1} \sigma\right)}{B_{2}^{2}+\left(B_{1} \sigma\right)^{2}}\right\},
$$

where for $\tau=0$, the steady state at $E_{0}$ was stable. When $\tau=\tau_{0}$, the characteristic equation has a pair of imaginary roots $\pm i \sigma$ where bifurcation will occur. As $\tau>\tau_{0}$, there was at least one root with positive real part.

\section{Tumour-Model in the Presence of Immune Response}

In this subsection, the effect of immune response was added to study how immune system can change the dynamical behaviour of tumour cell. When adding immune response $I(t)$, the system became

$$
\begin{aligned}
& x(t)=a_{1} z(t)+2 a_{3}(1-f) y(t)-a_{2} x(t)-d_{1} x(t)-c_{1} x(t) I(t), \\
& \mathrm{y}(t)=a_{2} x(t-\tau)-a_{3} y(t)-d_{2} y(t)-c_{2} y(t) I(t), \\
& z(t)=2 a_{3} f y(t)-a_{1} z(t)-d_{3} z(t)-c_{3} z(t) I(t), \\
& I(t)=k+\frac{\rho I(t)(x+y+z)^{n}}{\alpha+(x+y+z)^{n}}-d_{4} I(t)-c_{4} x(t) I(t)-c_{5} y(t) I(t)-c_{6} z(t) I(t) .
\end{aligned}
$$

Note that $E_{1}=\left(0,0,0, \frac{k}{d_{4}}\right)$ is an equilibrium point of this system with zero tumour level and a positive immune level. The Jacobian matrix about $E_{1}$ was

$$
A=\left[\begin{array}{cccc}
-a_{1}-d-c_{1} \frac{k}{d_{1}} & 2 a_{3}(1-f) & a_{1} & 0 \\
0 & -a_{3}-d_{2}-c_{2} \frac{k}{d_{1}} & 0 & 0 \\
0 & 2 a_{3} f & -a_{1}-d_{3}-c_{3} \frac{k}{d_{1}} & 0 \\
-c_{4} \frac{k}{d_{1}} & -c_{5} \frac{k}{d_{1}} & -c_{6} \frac{k}{d_{1}} & -d_{4}
\end{array}\right], B=e^{-\lambda \tau}\left[\begin{array}{cccc}
0 & 0 & 0 & 0 \\
a_{2} & 0 & 0 & 0 \\
0 & 0 & 0 & 0 \\
0 & 0 & 0 & 0
\end{array}\right] .
$$

Clearly, $\lambda=-d_{4}$ was an eigenvalue, and the remaining eigenvalues were given by the solutions to the characteristic equation

$$
\lambda^{3}+C_{1} \lambda^{2}+C_{2} \lambda+C_{3}+\left(D_{1} \lambda+D_{2}\right) e^{-\lambda \tau}=0,
$$


where

$$
\begin{aligned}
C_{1}= & a_{1}+a_{2}+a_{3}+d_{1}+d_{2}+d_{3}+c_{1} \frac{k}{d_{1}}+c_{2} \frac{k}{d_{1}}+c_{3} \frac{k}{d_{1}}, \\
C_{2}= & a_{1} a_{2}+a_{1} a_{3}+a_{2} a_{3}+a_{1} d_{1}+a_{3} d_{1}+a_{1} d_{2}+a_{2} d_{2}+d_{1} d_{2}+a_{2} d_{3}+a_{3} d_{3}+d_{1} d_{3}+d_{2} d_{3} \\
& +a_{1} c_{1} \frac{k}{d_{1}}+a_{3} c_{1} \frac{k}{d_{1}}+a_{1} c_{2} \frac{k}{d_{1}}+a_{2} c_{2} \frac{k}{d_{1}}+a_{2} c_{3} \frac{k}{d_{1}}+a_{3} c_{3} \frac{k}{d_{1}}+c_{2} d_{1} \frac{k}{d_{1}}+c_{3} d_{1} \frac{k}{d_{1}} \\
& +c_{1} d_{2} \frac{k}{d_{1}}+c_{3} d_{2} \frac{k}{d_{1}}+c_{1} d_{3} \frac{k}{d_{1}}+c_{2} d_{3} \frac{k}{d_{1}}+c_{1} c_{2}\left(\frac{k}{d_{1}}\right)^{2}+c_{1} c_{3}\left(\frac{k}{d_{1}}\right)^{2}+c_{2} c_{3}\left(\frac{k}{d_{1}}\right)^{2}, \\
C_{3}= & a_{1} a_{2} a_{3}+a_{1} a_{3} d_{1}+a_{1} a_{2} d_{2}+a_{1} d_{1} d_{2}+a_{2} a_{3} d_{3}+a_{3} d_{1} d_{3}+a_{2} d_{2} d_{3}+d_{1} d_{2} d_{3}+a_{1} a_{3} c_{1} \frac{k}{d_{1}} \\
& +a_{1} a_{2} c_{2} \frac{k}{d_{1}}+a_{2} a_{3} c_{3} \frac{k}{d_{1}}+a_{1} c_{2} d_{1} \frac{k}{d_{1}}+a_{3} c_{3} d_{1} \frac{k}{d_{1}}+a_{1} c_{1} d_{2} \frac{k}{d_{1}}+a_{2} c_{3} d_{2} \frac{k}{d_{1}}+c_{3} d_{1} d_{2} \frac{k}{d_{1}} \\
& +a_{3} c_{1} d_{3} \frac{k}{d_{1}}+a_{2} c_{2} c_{3} \frac{k}{d_{1}}+c_{2} d_{1} d_{3} \frac{k}{d_{1}}+c_{1} d_{2} d_{3} \frac{k}{d_{1}}+a_{1} c_{1} c_{2}\left(\frac{k}{d_{1}}\right)^{2}+a_{3} c_{1} c_{3}\left(\frac{k}{d_{1}}\right)^{2} \\
& +a_{2} c_{2} c_{3}\left(\frac{k}{d_{1}}\right)^{2}+c_{2} c_{3} d_{1}\left(\frac{k}{d_{1}}\right)^{2}+c_{1} c_{2} d_{2}\left(\frac{k}{d_{1}}\right)^{2}+c_{1} c_{2} d_{3}\left(\frac{k}{d_{1}}\right)^{2}+c_{1} c_{2} c_{3}\left(\frac{k}{d_{1}}\right)^{2}, \\
D_{1}= & -2(1-f) a_{2} a_{3}, \\
D_{2}= & -2(1-f) a_{1} a_{2} a_{3}-2(1-f) a_{2} a_{3} d_{3}-2 a_{1} a_{2} a_{3} f-2(1-f) a_{2} a_{3} c_{3} \frac{k}{d_{1}} .
\end{aligned}
$$

When there was no delay $(\tau=0)$, the characteristic Equation (7) became

$$
\lambda^{3}+C_{1} \lambda^{2}+\left(C_{2}+D_{1}\right) \lambda+\left(C_{3}+D_{2}\right)=0 .
$$

The steady state was seen stable in the absence of delay if the roots of the characteristic Equation (8) have negative real parts. Applying Routh-Hurwitz Criteria, the necessary and sufficient conditions for the system to be stable was

1. $C_{1}>0$.

2. $C_{3}+D_{2}>0$.

3. $C_{1}\left(C_{2}+D_{1}\right)>\left(C_{3}+D_{2}\right)$.

Now, the effect of delay was added in the system. When the delay was positive $(\tau>0)$, the characteristic equation became as in (7). This study followed the step as in the case for tumour model in the absence of immune response and the critical value $\tau_{0}$ was defined by

$$
\tau_{0}=\frac{1}{\sigma}\left\{\frac{-\left(D_{2}\right)\left(-C_{1} \sigma^{2}+C_{3}\right)-\left(-\sigma^{3}+C_{2} \sigma\right)\left(D_{1} \sigma\right)}{D_{2}^{2}+\left(D_{1} \sigma\right)^{2}}\right\},
$$

where for $\tau=0$, the steady state at $E_{1}$ was stable. When $\tau=\tau_{0}$, the characteristic equation has a pair of imaginary roots $\pm i \sigma$ where bifurcation will occur. As $\tau>\tau_{0}$, there was at least one root with positive real part. 


\section{Simulation Results}

In this section, original model (6) was used to determine the results simulation for tumour population. The result of the stability was tested using a specific set of parameters as in [1314]. DDE23 solver in MATLAB was used to validate the stability conditions for the proposed model.

For the case without immune response, the simulation for model (1) was shown as in Figure 2. The graph shows that the tumour grows exponentially for every phases. As the population of tumour cells that enter quiescent phase, $f$, increases, the population of tumour cells in an active cycle (interphase and mitosis phase) decreased. When $f=0.1$, the population of tumour cell at quiescent phase was the lowest and population of tumour cell at mitosis was the highest. This happened when the tumour cell enters the active part of the cycle and successfully passed interphase and go to mitosis. Then, $f$ was set to 0.9 . The highest population of tumour cell happened in mitosis and the lowest happened during interphase. From both of figures, it can be seen that the population of the tumour cells decreased at every phases of the cell cycle when the population of tumour cells that enter $G_{0}$ phase increased. This showed that the rate of proliferation of tumour cells is depending on the population of tumour cells that enter $G_{0}$ phase.

When immune response was added to the model, the result for model (6) showed that the population of tumour cells started to decrease in every phases of the cell cycle (Figure 4). From the higher values, the graph decreased gradually and indicated that the tumour cells start to eradicate. This means that in this case, a small immune response is able to kill the tumour cells. The population of immune response also decreased and at the certain time, it became stable to the steady state.

A time delay, $\tau$ was applied to both models (1) and (6). The tumour equilibrium showed that the tumour free equilibrium was still unstable for model (1) and keep stable for model (6) as shown in Figure 5 and 6. However, the tumour had grown much slower with time delay model compared to that without delay.

\section{Conclusion}

The presented model has represented the interaction between tumour cells and immune response based on the dynamics of the cell cycle. The relation between the cell cycle and tumour was obvious; cell cycle controls cell proliferation, and tumour is a disease of inappropriate cell proliferation. Cell cycle consists of active and inactive phases. In this model, both phases have been considered. Compared to Liu's model, it was assumed that a new daughter cell from mitosis will enter either inter phase or quiescent phase. This is more relevant to the real process of cell cycle. By this assumption, the result had showed that the population of tumour cells mainly depends on natural death for every phases and number of tumour cells that enter quiescent phase. Tumour cells that enter quiescent phase is called dormant cell and less sensitive to cancer treatments. At the certain time, the dormant cell will enter the cell cycle and restart tumour growth. Then, more treatments are needed to eradicate the tumour growth. The inclusion of quiescent phase provided a deep sight into the cancer development and helped to understand how dormant population contribute in diseases.

Besides, the model has also considered a basic interaction between tumour cell and immune 


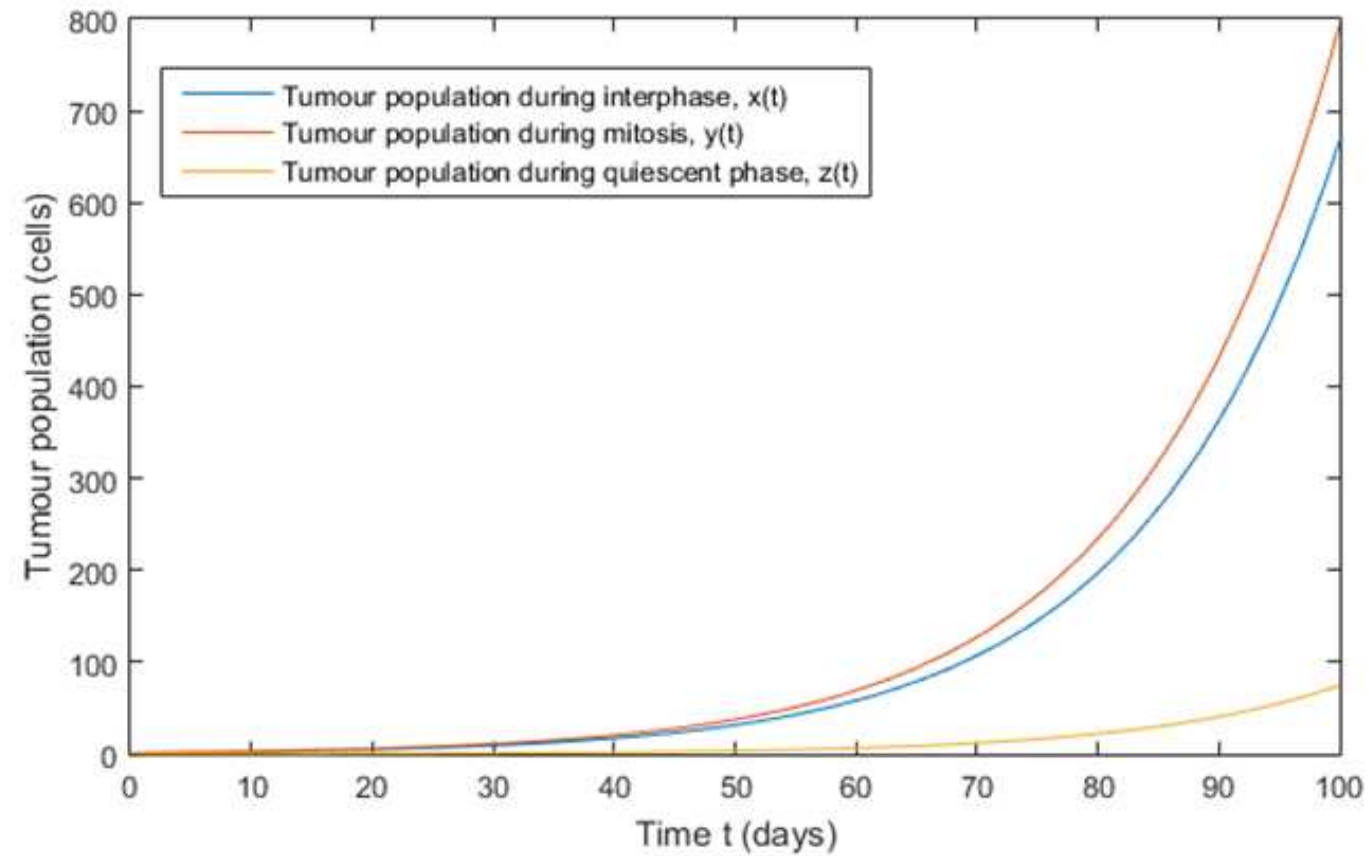

(a)

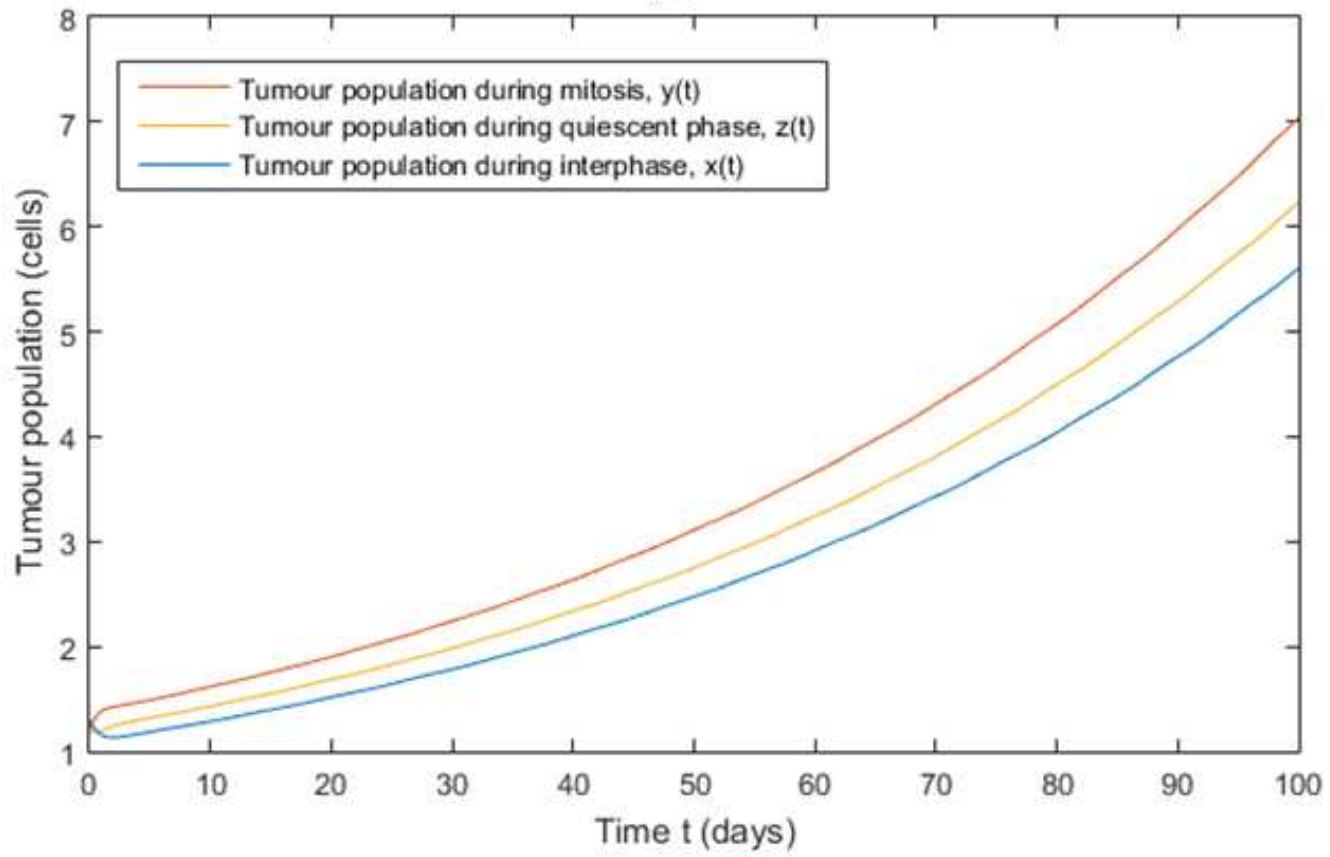

(b)

Figure 3: Tumour Population at Interphase, Mitosis and Quiescent Phases in the Absence of Immune Response Based on Different Values of $f$; (a) $f=0.1$ and (b) $f=0.9$; with the Initial Condition $x(0)=1.3, y(0)=1.2$ and $z(0)=1.2$, respectively 


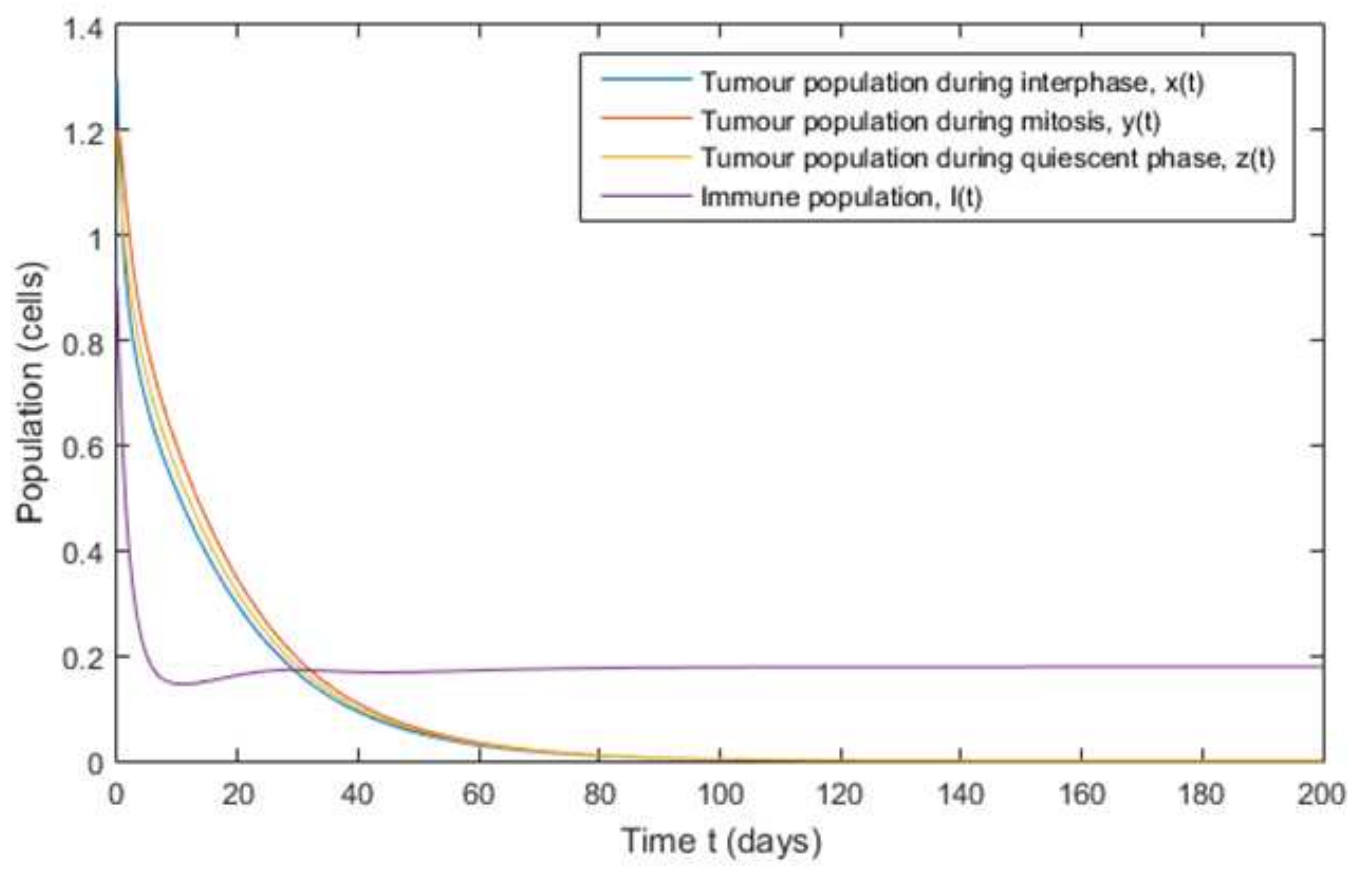

Figure 4: Tumour Population at Interphase, Mitosis and Quiescent Phases with Immune

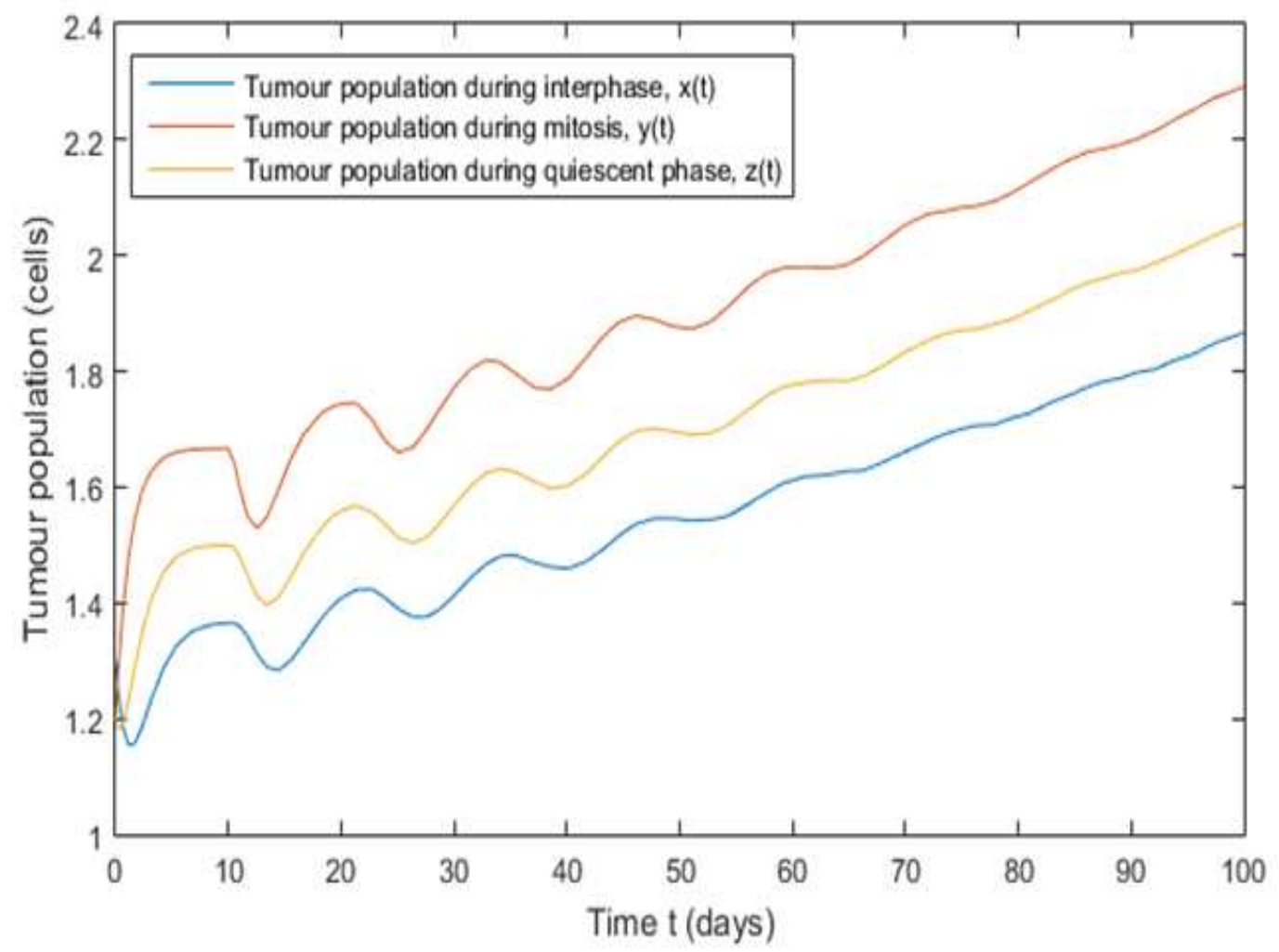

Figure 5: Tumour Population at Interphase, Mitosis and Quiescent Phases without Immune Response when $\tau=10$ 


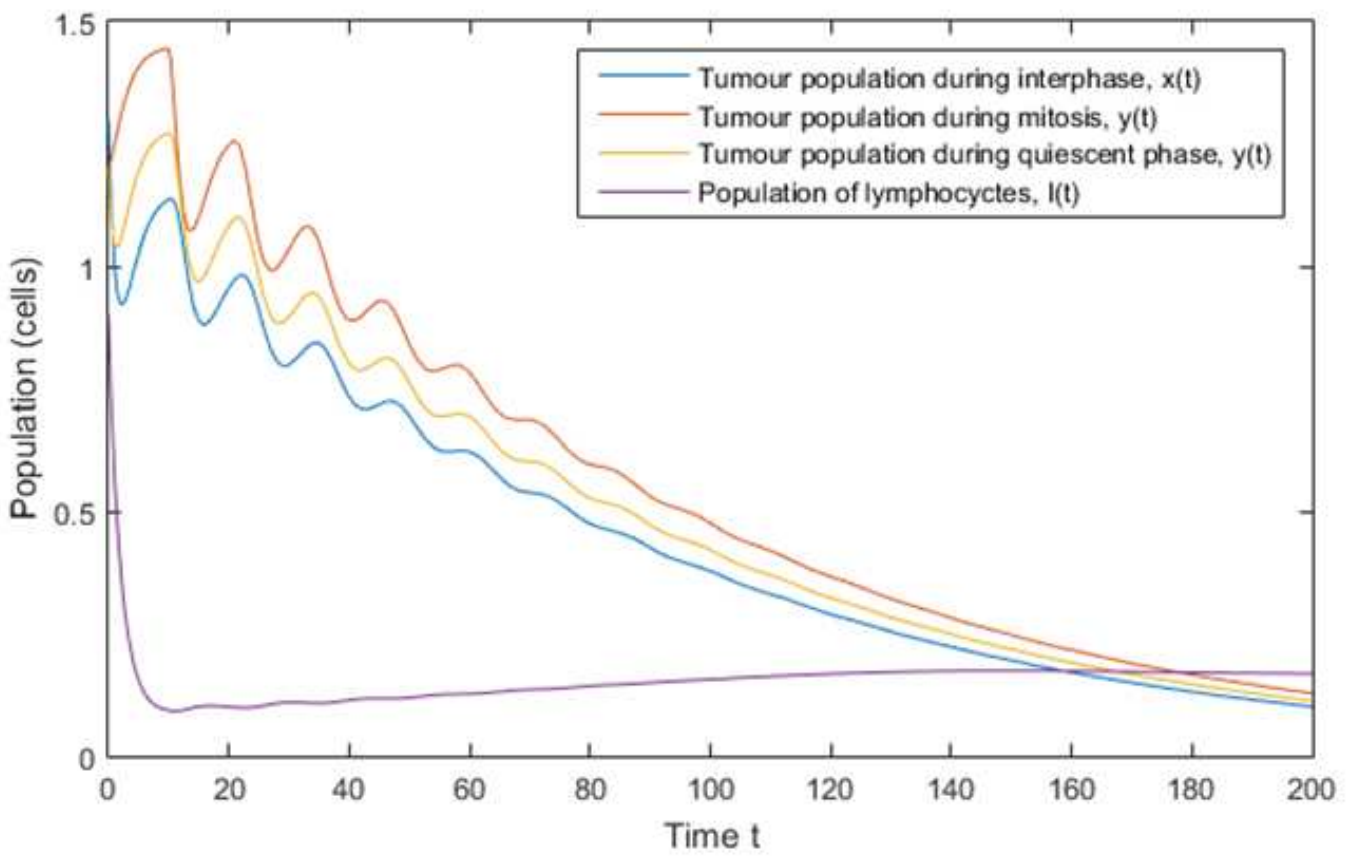

Figure 6: Tumour Population at Interphase, Mitosis and Quiescent Phases with Immune Response when $\tau=10$

system. Immune system is a complex system and this study only considered the general interaction with the tumour cell. Adding immune responses in the model has greatly helped to stabilise the system and inhibit further growth of tumour cells. In small cases, immune system is able to clear the tumour cell and prevent the tumour recurrence.

\section{Acknowledgement}

The authors are thankful to Research Management Centre (UTM) and Ministry of Education Malaysia for financial support with vote numbers 07397, 4L854 and 16H26.

\section{References}

[1] Jemal, A., Bray, F., Center, M. M., Ferlay, J., Ward, E. and Forman, D. Global cancer statistics. CA: a Cancer Journal for Clinicians. 2011.61(2): 69-90.

[2] Stewart, B. and Wild, C. P. World Cancer Report 2014. World. 2016.

[3] Kirschner, D., and Panetta, J. C. Modeling Immunotherapy of the tumor-immune interaction. Journal of Mathematical Biology. 1998.37(3): 235-252.

[4] De Pillis, L. G., and Radunskaya, A. A mathematical tumor model with immune resistance and drug therapy: an optimal control approach. Computational and Mathematical Methods in Medicine. 2001. 3(2): 79-100.

[5] Awang, N. A., and Maan, N. Analysis of tumor populations and immune system interaction model. AIP Conference Proceedings. 2016. 1750(1): 030049. 
[6] Eguizabal, C., Zenarruzabeitia, O., Monge, J., Santos, S., Vesga, M. A., Maruri, N. and Borrego, F. Natural killer cells for cancer immunotherapy: pluripotent stem cells-derived NK cells as an immunotherapeutic perspective. Frontiers in Immunology. 5. 2014.

[7] Fruci, D., Lo, M. E., Cifaldi, L., Locatelli, F., Tremante, E., Benevolo, M., and Giacomini, P. T.NK cells: two sides of tumor immunoevasion. J. Transl. Med. 2013. 11: 30.

[8] Eladdadi, A., and Radunskaya, A. E. Modeling cancer-immune responses to therapy. Journal of Pharmacokinetics and Pharmacodynamics. 2014. 41(5): 461-478.

[9] de Pillis, L. G., Radunskaya, A. E., and Wiseman, C. L. A validated mathematical model of cell-mediated immune response to tumor growth. Cancer Research. 2005. 65(17): 79507958.

[10] d'Onofrio, A. A general framework for modeling tumor-immune system competition and immunotherapy: mathematical analysis and biomedical inferences. Physica D: Nonlinear Phenomena. 2005. 208(3): 220-235.

[11] Yafia, R. A study of differential equation modeling malignant tumor cells in competition with immune system. International Journal of Biomathematics. 2011. 4(02): 185-206.

[12] Cheng, M., Chen, Y., Xiao, W., Sun, R., and Tian, Z. NK cell-based immunotherapy for malignant diseases. Cellular and Molecular Immunology. 2013. 10(3): 230-252.

[13] Villasana, M., and Radunskaya, A. A delay differential equation model for tumor growth. Journal of Mathematical Biology. 2003. 47(3): 270-294

[14] Liu, W., Hillen, T., and Freedman, H. A mathematical model for M-Phase specific chemotherapy including the G0-Phase and immunoresponse. Mathematical Biosciences and Engineering. 2007. 4(2): 239.

[15] Hillen, T., de Varies, G., Gomg, J. and Finlay, C. From cell population models to tumor control probability: including cell cycle effects. Acta Oncologica. 2010. 49(8): 1315-1323.

[16] Watzl, C., Sternberg-Simon, M., Urlaub, D., and Mehr, R. Understanding natural killer cell regulation by mathematical approaches. Frontiers in Immunology. 2013. 3. 